\title{
ADAMTS19-associated heart valve defects: Novel genetic variants consolidating a recognizable cardiac phenotype
}

\author{
Salam Massadeh $^{1,2} \quad$ | Amal Alhashem ${ }^{3,4}$ | Ingrid M.B.H. van de Laar ${ }^{5}$ (1) | \\ Fahad Alhabshan $^{6}$ | Natalia Ordonez ${ }^{7}$ | Salem Alawbathani ${ }^{7}$ | Suliman Khan ${ }^{7}$ | \\ Mohamed S. Kabbani $^{6}$ | Farah Chaikhouni ${ }^{6}$ | Atia Sheereen ${ }^{1}$ | Iman Almohammed ${ }^{1,2}$ | \\ Bader Alghamdi $^{1}$ | Ingrid Frohn-Mulder ${ }^{8}$ | Salim Ahmad ${ }^{9}$ | Christian Beetz ${ }^{7}$ (1) | \\ Peter Bauer $^{7}$ ( ) | Marja W. Wessels ${ }^{5}$ | Manal Alaamery ${ }^{1,2}$ | Aida M. Bertoli-Avella ${ }^{7}$ ()
}

\begin{abstract}
${ }^{1}$ Department of Developmental Medicine, King Abdullah International Medical Research Center, King Saud Bin Abdulaziz University for Health Sciences, King Abdulaziz Medical City, Ministry of National Guard Health Affairs, Riyadh, Saudi Arabia

${ }^{2}$ The Joint Center of Excellence for Biomedicine Between King Abdulaziz City for Science and Technology (KACST) and Brigham \& Women's Hospital (BWH), Joint Centers of Excellence Program, King Abdulaziz City for Science and Technology (KACST), Riyadh, Saudi Arabia

${ }^{3}$ Division of Pediatric Genetics, Department of Pediatrics, Prince Sultan Military Medical City, Riyadh, Saudi Arabia

${ }^{4}$ Department of Anatomy and Cell biology, College of Medicine, Alfaisal University, Riyadh, Saudi Arabia

${ }^{5}$ Department of Clinical Genetics, Erasmus University Medical Center, Rotterdam, The Netherlands

${ }^{6}$ Department of Cardiac Sciences, Ministry of the National Guard-Health Affairs, King Abdullah International Medical Research Center, King Saud bin Abdulaziz University for Health Sciences, Riyadh, Saudi Arabia

${ }^{7}$ CENTOGENE AG, Rostock, Germany

${ }^{8}$ Department of Pediatric Cardiology, Erasmus University Medical Center, Rotterdam, The Netherlands

${ }^{9}$ Prince Sultan Cardiac Center, Prince Sultan Military Medical City, Riyad, Saudi Arabia
\end{abstract}

\section{Correspondence}

Aida M. Bertoli-Avella, CENTOGENE AG. Am Strande 7, 18055, Rostock, Germany. Email: aida.bertoli-avella@centogene.com

Funding information

King Abdullah International Medical Research Center, Grant/Award Number: RC15/140

\section{Abstract}

Recently, ADAMTS19 was identified as a novel causative gene for autosomal recessive heart valve disease (HVD), affecting mainly the aortic and pulmonary valves. Exome sequencing and data repository (CentoMD) analyses were performed to identify patients with ADAMTS19 variants (two families). A third family was recognized based on cardiac phenotypic similarities and SNP array homozygosity. Three novel loss of function (LoF) variants were identified in six patients from three families. Clinically, all patients presented anomalies of the aortic/pulmonary valves, which included thickening of valve leaflets, stenosis and insufficiency. Three patients had (recurrent) subaortic membrane, suggesting that ADAMTS19 is the first gene identified related to discrete subaortic stenosis. One case presented a bi-commissural pulmonary valve. All patients displayed some degree of atrioventricular valve insufficiency. Other cardiac anomalies included atrial/ventricular septal defects, persistent ductus arteriosus, and mild dilated ascending aorta. Our findings confirm that biallelic LoF variants in ADAMTS19 are causative of a specific and recognizable cardiac phenotype. We recommend considering ADAMTS19 genetic testing in all patients with multiple semilunar valve abnormalities, particularly in the presence of subaortic membrane. ADAMTS19 screening in patients with semilunar valve abnormalities is needed to estimate the frequency of the HVD related phenotype, which might be not so rare.

\section{KEYWORDS}

ADAMTS19, aortic valve stenosis, pulmonary stenosis, subaortic membrane 


\section{1 | INTRODUCTION}

The human heart is one of the first organs to function during embryonic life. In fact, proper function of the cardiovascular system is critical for embryonic survival. ${ }^{1}$ The complex stages of cardiovascular morphogenesis include heart tube formation and looping followed by chamber formation, remodeling and formation of the arterial trunks. ${ }^{1,2}$ The process requires a delicate balance that if perturbed, usually leads to heart defects. $^{1}$

Congenital heart disease (CHD) is the most common form of structural birth defects observed in humans, ${ }^{3}$ with bicuspid aortic valve (BAV) being the most common $\mathrm{CHD} .^{4}$ BAV may be asymptomatic and undetected in early life but poses a risk for serious complications and sudden cardiac death later in life. ${ }^{5}$ The extreme clinical variability, reduced penetrance, and suspected genetic heterogeneity have complicated the identification of genes involved in non-syndromic CHD, with only a limited number of genes identified to date. ${ }^{6-9}$

Recently, Wünnemann et al identified biallelic loss of function (LoF) variants in ADAMTS19 as causative for heart valve disease (HVD), affecting mainly the aortic and pulmonary valves as described in four patients from two families. ${ }^{10}$ Furthermore, $38 \%$ of homozygous Adamts 19 knockout mice presented progressive aortic valve disease, characterized by aortic valve stenosis and insufficiency. Histological examination of the dysfunctional aortic valve from knockout mice showed disorganization of the extracellular matrix (ECM), with thickened valve leaflets and collagen deposition. ${ }^{10}$ Single cell RNA-sequencing (scRNA) identified the Klf2 shear stress signature, with apparent valvular interstitial cells to endocardial signaling. The authors concluded that loss of Adamts19 perturbs shear stress signaling in endothelial cells of the aortic valve, which in time leads to increased cellularity and proteoglycan deposition in the valves, ECM disorganization and HVD. ${ }^{10}$

Here we present three additional families with an autosomal recessive HVD primarily affecting the semilunar valves and novel, homozygous, presumed LoF variants in ADAMTS19. Our findings confirm the causative role of ADAMTS19 variants for congenital HVD and consolidate the related human cardiac phenotype.

\section{2 | METHODS}

All five family members (index, parents, affected and unaffected siblings) from family 1 , the index patient from family 2 and index and affected sibling from family 3 were exome sequenced at CENTOGENE AG (Rostock, Germany), as previously described. ${ }^{11}$ Libraries were sequenced on an Illumina Hiseq 4000 with 150 bp paired end sequencing. Variant calling was performed using GATK. Variants with a phred-scaled quality score $>215^{12}$ and a MAF of $<1 \%$ were retained for analysis. Variants were further filtered based on zygosity and segregation in the family, with the assumption that a causal variant was segregating in an autosomal recessive manner (heterozygous in parents, homozygous or compound heterozygous in affected offspring and wild type or heterozygous in the unaffected sibling). For family 3 , only ADAMTS19 and genes from a CHD panel were evaluated (CFC1, CITED2, CRELD1, FOXH1, GATA4, GATA6, GDF1, NKX2-5, NOTCH1, TBX1, TBX20, ZFPM2).
Our database CentoMD ${ }^{13}$ was queried for rare variants $(<1 \%)$ in ADAMTS19 (9 September 2019) and the resulting data was filtered taking into consideration an autosomal recessive mode of inheritance and the impact of the variant (LoF were prioritized).

The variant-containing exons of ADAMTS19 (NM_133638.4) were amplified (primers available upon request) and Sangersequenced from both sides on a 3730xl sequencer (Thermo Fisher Scientific, Waltham, Massachusetts) in all available family members for co-segregation analysis.

Informed written consents were obtained from patients' parents and referring clinicians. All studies were performed within the exome sequencing diagnostic procedures.

\section{RESULTS}

\section{$3.1 \mid$ Family 1}

The index patient is a 7-year-old female born to healthy, consanguineous (second-cousins) parents, with no relevant family medical history. She was born after an uneventful full-term pregnancy with normal delivery. During her first year of life, she had recurrent respiratory infections and around the age of 10 months, a murmur was noticed during pediatric consultation. Echocardiography showed thickened and doming aortic valve leaflets and subaortic membrane (SAM). She underwent surgical resection of the SAM at the age of 16 months. She had recurrent SAM that required a re-intervention at the age of 3 years. Recently, echocardiography showed again a recurring SAM with very thickened and doming aortic valve leaflets (tri-commissural with partial commissural fusion), mild aortic insufficiency, and severe left ventricular outflow tract (LVOT) obstruction. She had doming pulmonary valve leaflets (bi-commissural) with mild insufficiency. Additionally, she had severe left ventricular hypertrophy with good biventricular systolic function. Currently she has mild intermittent symptoms mainly consisting of chest pain during excessive playing. Her psychomotor development is appropriated for her age. She is currently under regular follow-up by the cardiologist. A future cardiac intervention is planned (Ross-Kono procedure).

Her brother is a 4-year-old boy who was found to have SAM and ventricular septum defect (VSD) since birth. The VSD closed spontaneously. He then received a follow-up for the SAM and LVOT obstruction. Echocardiography showed a small SAM, moderate aortic valve stenosis, mild aortic valve insufficiency, trivial tricuspid valve insufficiency, and good left ventricular systolic function. No other abnormalities were reported. Surgical repair (SAM resection) was performed successfully at the age of 3 years. He is clinically asymptomatic with good activity tolerance. Figure 1A1, C summarizes the genealogical tree and echocardiography findings in this family.

\subsection{Family 2}

The index case is a 9-year-old female born to consanguineous parents. There is family history of short stature. She was born prematurely at 34 weeks of gestation by emergency cesarean section because of 
$\left(A_{1}\right)$

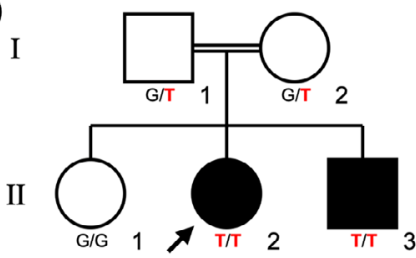

$\left(A_{2}\right)$

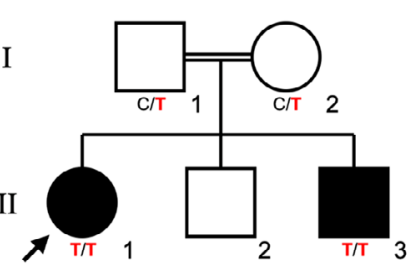

$\left(A_{3}\right)$

I

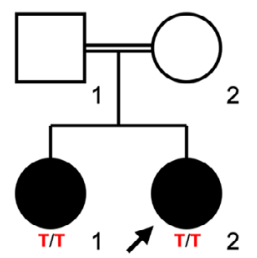

$\left(\mathrm{B}_{1}\right)$

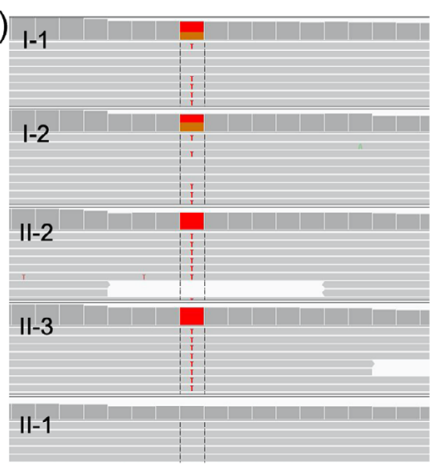

$\left(\mathrm{B}_{2}\right)$

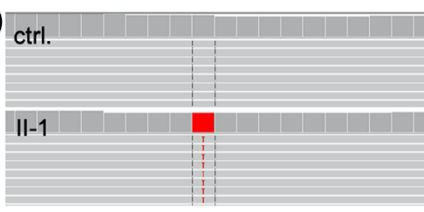

ctrl.

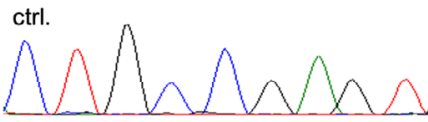

|l-1

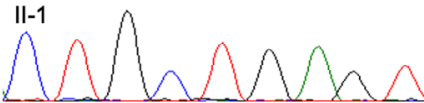

$\left(\mathrm{B}_{3}\right)$

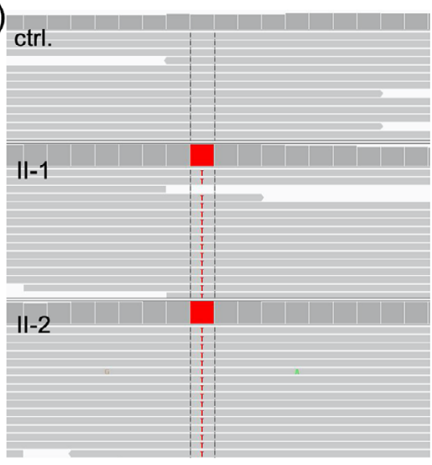

(C)
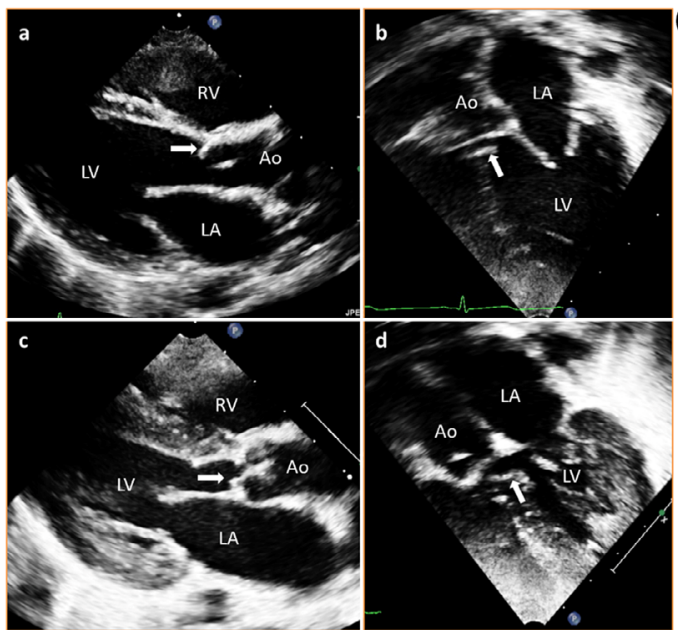

(D) a
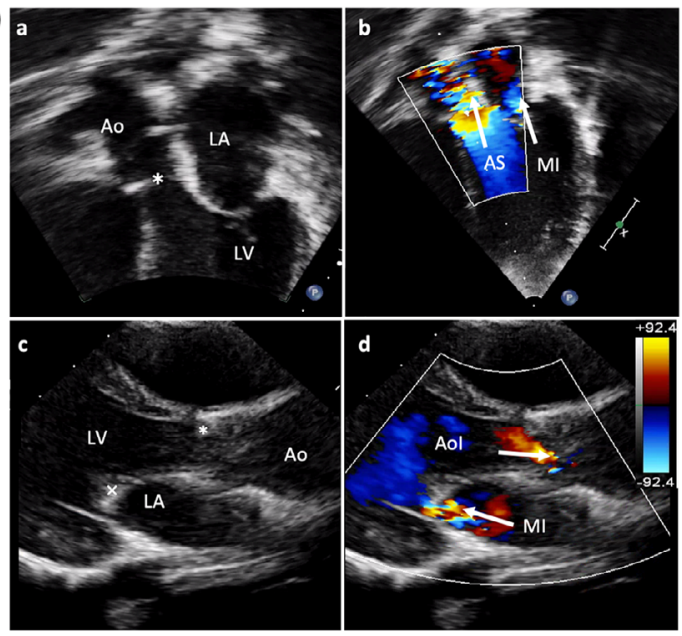

(E)

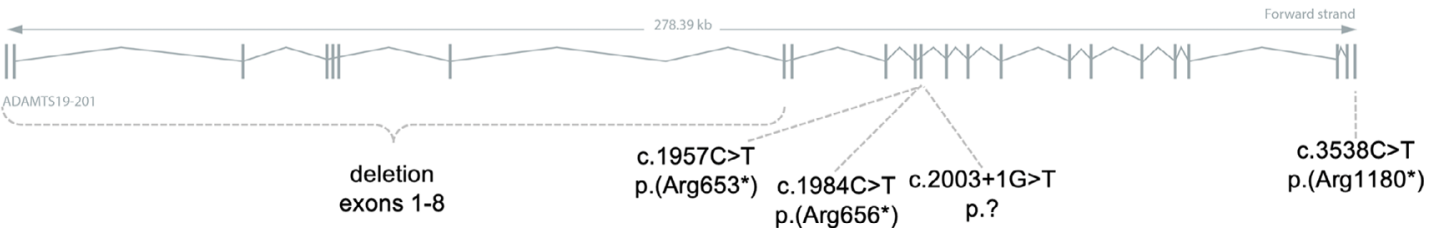

FIGURE 1 Family trees, and associated ADAMTS19 genotypes showing full co-segregation with the cardiac phenotype. All patients are homozygotes for LoF variants in ADAMTS19. LoF, loss of function. A1, Family 1 pedigree with parents, healthy sibling, index and brother included. B1, Corresponding Integrative Genomics Viewer (IGV) ${ }^{14}$ images are shown (NM_133638.4(ADAMTS19):c.2003+1G>T). C, Echocardiography views in long axis and five chambers planes for the brother $(a, b)$ and the index $(c, d)$ showing the presence of SAM (arrow) under the aortic valve. Ao, aorta; LA, left atrium; LV, left ventricle; RV, right ventricle. A2, Family 2 pedigree with parents, index and affected sibling included. B2, IGV images from the index and a control individual and Sangers traces for index and a control individual are shown (NM_133638.4(ADAMTS19): c.3538C > T, p.Arg1180*). A3, Family 3 pedigree with parents (not tested), index and affected sister. B3, IGV images from index, affected sibling and a control individual (NM_133638.4(ADAMTS19):c.1957C>T, p.(Arg653*)). D, Index 2D echocardiography, apical view, detailed on valvular apparatus *:subvalvular aortic stenosis (a). Color Doppler echocardiography, showing disturbances of the aortic flow. AS, aortic stenosis; MI, mitral insufficiency (b). Affected sister: 2D echocardiography, parasternal long axis. Ao, dilated ascending aorta. *: subvalvular aortic stenosis; $x$, thickened mitral valve (a). Color doppler echocardiography. Aol, aortic insufficiency (b). E, Schematic representation of ADAMTS19 gene (following Ensemble genome browser ${ }^{15}$ ) and identified causative variants by Wünnemann et al and the current study [Colour figure can be viewed at wileyonlinelibrary.com]

intrauterine growth retardation (IUGR) and fetal distress. She was admitted to the neonatal intensive care unit given her prematurity, IUGR, and periventricular leukomalacia. She was found to have a heart murmur and diagnosed with severe pulmonary valve stenosis and small atrial septal defect (ASD) secundum with tiny persistent ductus arteriosus (PDA). She had delayed motor milestones; at 7 months old, she was not able to sit 
with support. However, at the age of one, she was able to stand and walk with support and spoke six to seven words. During a physical examination scaphocephaly, short and upslanting palpebral fissures were noticed. Currently, she has normal neurodevelopment and remains asymptomatic. The renal ultrasound was normal. The brain MRI showed delayed myelination and minimal cerebral atrophic changes. The skeletal survey showed scaphocephaly, normal bone density and delayed bone age. A chromosomal microarray (CMA) was performed with normal results.

Her younger brother was referred at age of $5 \frac{1}{2}$ months, because of dysmorphic facial features and CHD. He was born at term by a cesarean section. He displayed feeding difficulties from birth. A small ASD secundum and mild pulmonary stenosis were detected. He also had laryngeal cleft type 1 and bronchial asthma. He presented neurodevelopmental delay and autism spectrum disorder. At his last examination at 2 years old, he had speech delay (able to say only two words) and just started to walk. CMA was performed and showed a pathogenic deletion at chromosome 16p11.2 (1 copy loss) consistent with the chromosome $16 \mathrm{p} 11.2$ microdeletion syndrome. The deletion occurred de novo (both parents had normal CMA). Figure 1A2 shows the summarized genealogical tree of family 2 .

\subsection{Family 3}

The index is an 11-year-old female born to consanguineous parents. Medical family history is positive; a sister of the mother of the index was diagnosed with an unspecified congenital heart malformation. The index was born at 38 weeks gestation after an uneventful pregnancy and delivery, with a birthweight of $2755 \mathrm{~g}$. In the first days, feeding problems occurred, and 3 days after birth a heart murmur was noticed. Echocardiography revealed a severe pulmonary valve stenosis due to a small pulmonary valve orifice with dysplastic and thickened pulmonary valve leaflets. The right ventricle showed hypertrophy. Also, a milder valvular aortic stenosis was observed with thickened atrioventricular valves with mild insufficiency (mitral and tricuspid valve). A patent foramen ovale (PFO) with left to right shunting was present. Despite balloon valvuloplasty at the age of 3 months a substantial pulmonary valve stenosis remained. At the age of 2 years, a severe subaortic valve stenosis was observed, with moderate aortic valve insufficiency. At this time, the child had no physical complaints. At the age of 6 , complaints of fatigue were present and pulmonary valve commissurotomy was performed with resection of a SAM and closure of the PFO. After surgery a mild valvular and subvalvular aortic stenosis, mild aortic valve insufficiency, and moderate pulmonary valve stenosis with mild insufficiency remained without ventricular hypertrophy and normal right and left ventricular systolic function.

The second female child was born at term after an uneventful pregnancy and delivery with a birthweight of 3460g. She was diagnosed with severe pulmonary valve stenosis and mild aortic valve stenosis and insufficiency. At the age of 1 month, a successful balloon valvuloplasty of the pulmonary valve was performed. At the age of 5 , a mildly dilated ascending aorta $(23 \mathrm{~mm}, Z$-score+3.6) was present. Figure1A3 shows the summarized genealogical tree of the family and echocardiography findings (Figure 1D).
CMAs were performed in both affected siblings, including SNP array analyses. In both girls, a maternally inherited $1074 \mathrm{~kb}$ duplication on band 3p12.3 and a maternally inherited $246 \mathrm{~kb}$ deletion on band $6 \mathrm{q} 12$ were present. Both copy number variations were of unknown clinical significance. Large regions of copy-neutral homozygosity were observed in both siblings. NGS-based analysis of a panel of genes related to CHD resulted negative.

A summary of the most relevant clinical features is presented in Table 1.

\section{4 | Genetic findings}

In two families, exome sequencing was requested given the suspicion of an underlying autosomal recessive condition. Exome analysis did not reveal any diagnostic candidate variant. However, in family 1 an extended search beyond approved "diagnostic genes" detected a homozygous variant in a candidate gene (ADAMTS19). The gene is not described in OMIM as related to any phenotype (accessed January 9, 2020). Recently, LoF variants in the ADAMTS19 gene were described as causing non-syndromic HVD, consisting of thickened pulmonary and aortic valves, causing valve stenosis and insufficiency. ${ }^{10}$ In family 1 , the detected variant was affecting the canonical splicing site (NM_133638.4:c.2003+1G>T, p.?) and was found to co-segregate with the HVD phenotype (Figure 1A1,B1). This splice donor variant is predicted to lead to either skipping of the associated exon 12 or read through into the intron. Both scenarios result in frameshifts and LoF of the ADAMTS19 allele.

We searched our database CentoMD ${ }^{13}$ for rare, presumed LoF variants in ADAMTS19 and identified one additional patient with a novel homozygous variant in ADAMTS19(Figure 1A2). In this family (family 2), the initial exome sequencing request resulted negative (2018). Upon database queried, a homozygous nonsense variant (NM_133638.4: c. $3538 \mathrm{C}>\mathrm{T}$, p.Arg1180*) was detected in the index case (Figure 1B2). Carrier testing in parents and the affected sibling confirmed cosegregation of the variant with the cardiac phenotype (homozygous in index and affected sibling and heterozygous in parents, Figure 1A2,B2).

Family 3 was identified independently via collaboration with the department of Clinical Genetics (Erasmus University Medical Centre, the Netherlands). Both patients from family 3 were selected for ADAMTS19 sequencing based on their specific HVD phenotype, and shared region of homozygosity on chromosome 5 comprising the ADAMTS19 gene. Subsequently, targeted NGS analysis of the gene revealed a homozygous LoF variant, NM_133638.4:c.1957C>T, p.(Arg653*) in both affected individuals (Figure $1 \mathrm{~A} 3, \mathrm{~B} 3$ ).

All three variants were absent from gnomAD (last accessed January 9,2020 ) and detected for the first time in homozygous state in the CentoMD database. Disease causing ADAMTS19 variants reported to date are summarized in Figure 1E.

\section{DISCUSSION}

In this study, we present further evidence confirming that biallelic LoF ADAMTS19 variants cause a CHD affecting mainly the semilunar 


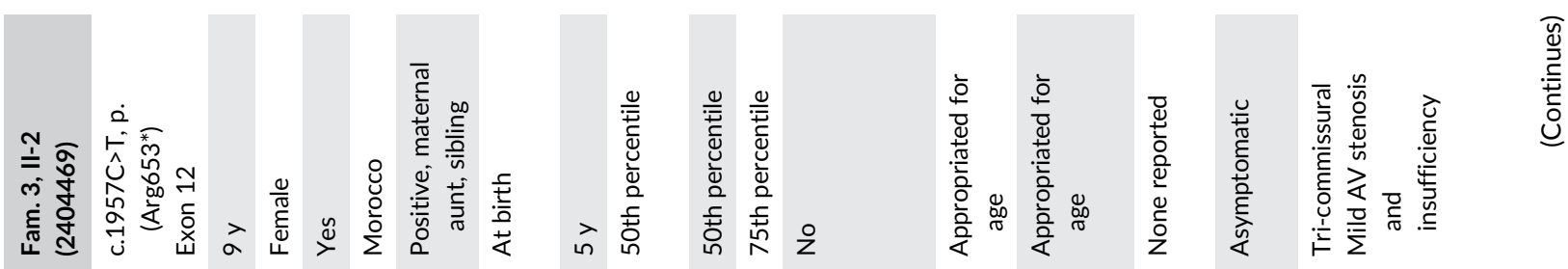

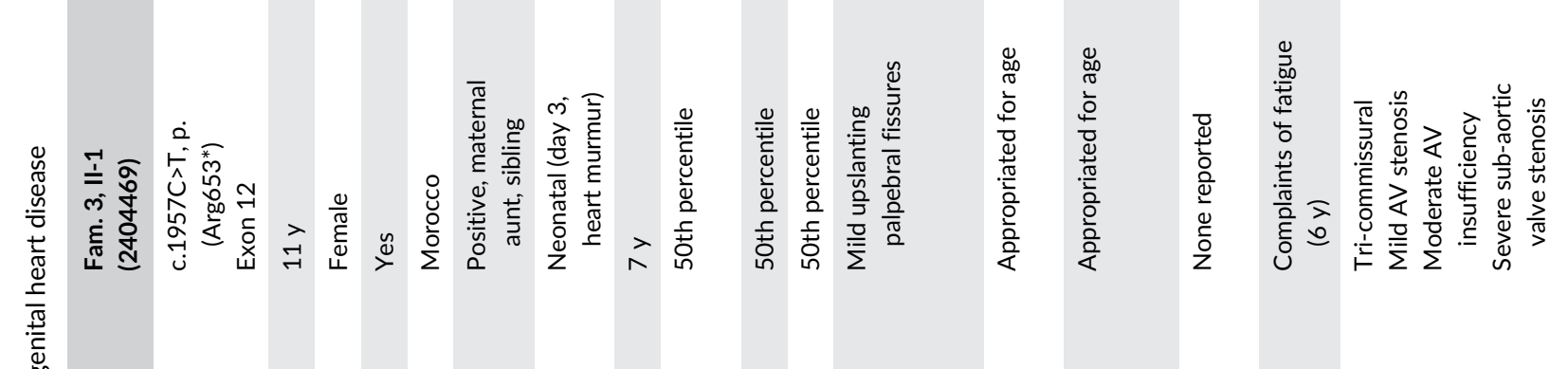

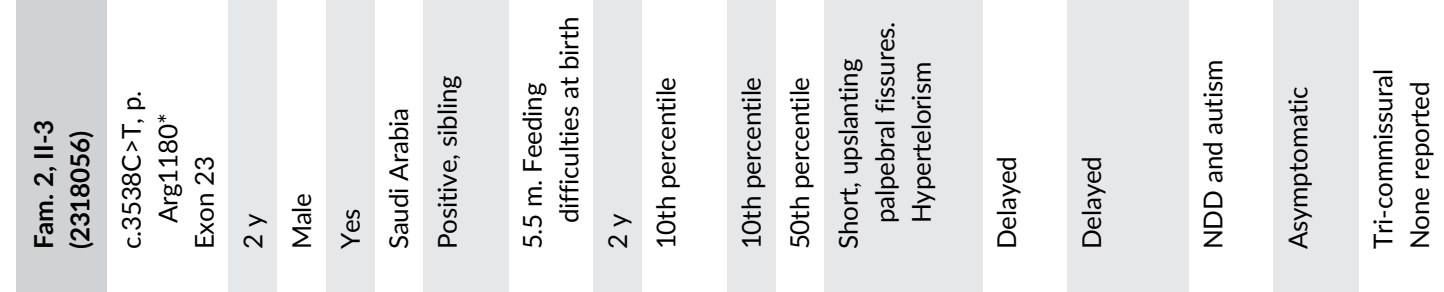

黄

旡

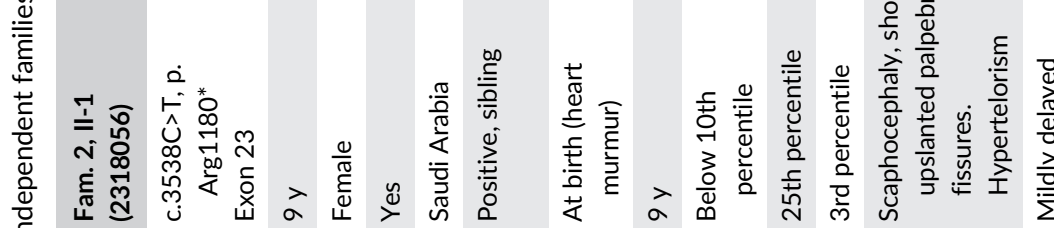

बे

进

$\stackrel{\varepsilon}{\stackrel{0}{\leftarrow}}$

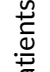

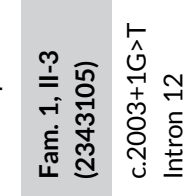

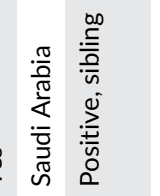

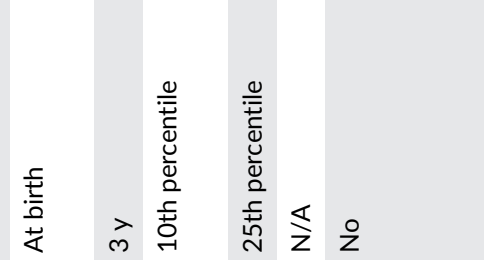

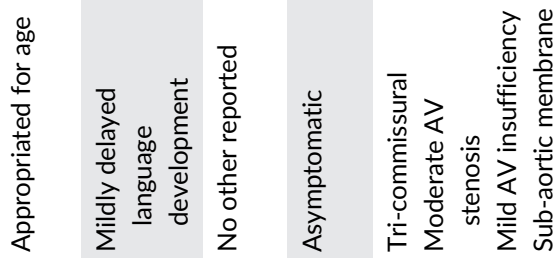

要

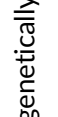

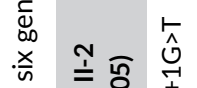

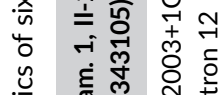
(1)

产 范

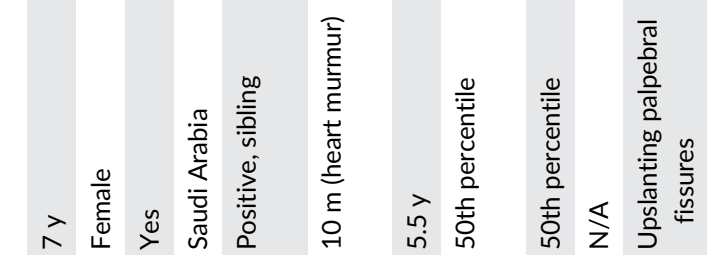

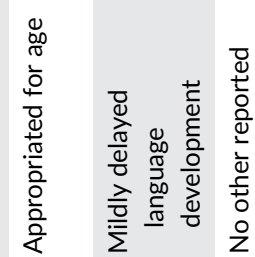

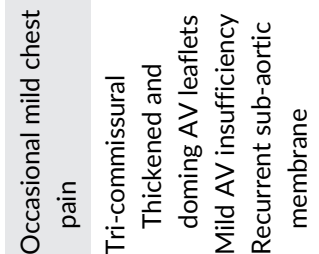

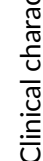

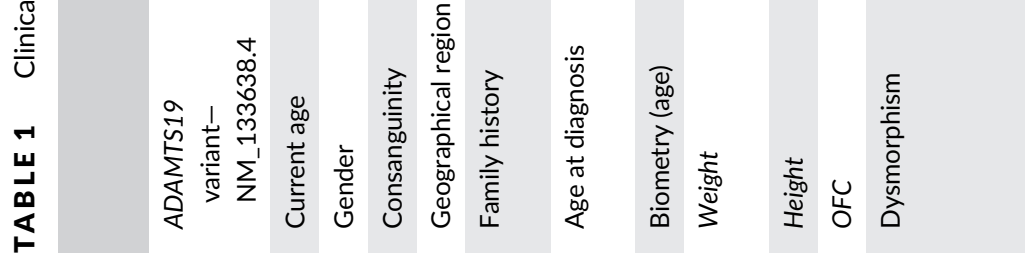

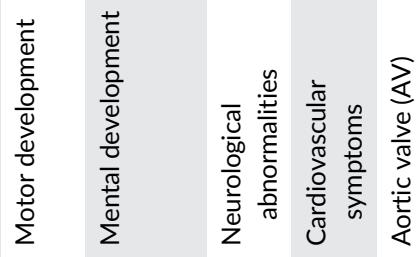




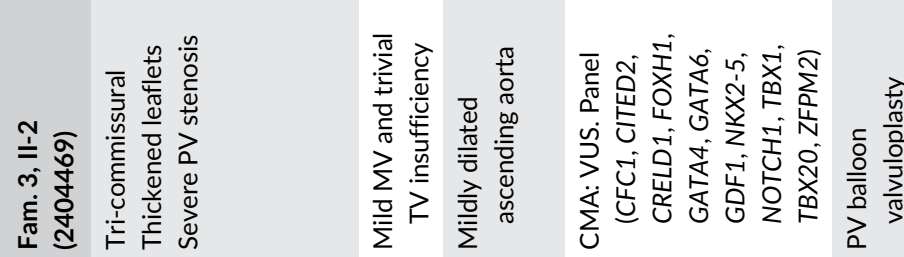

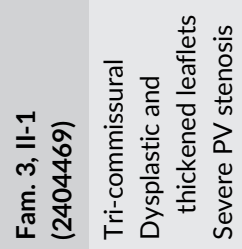

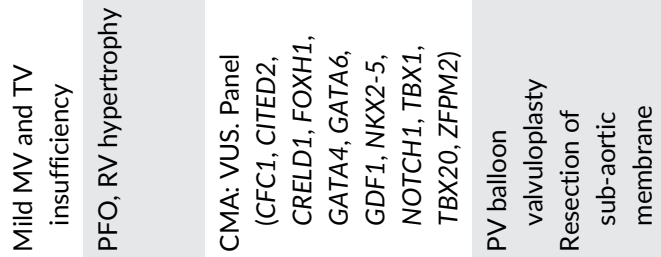

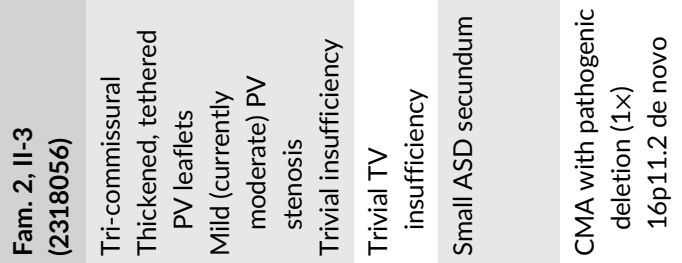

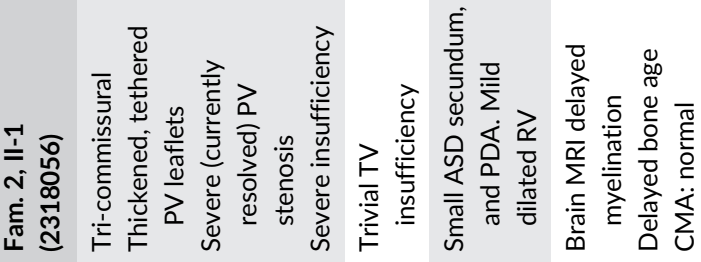

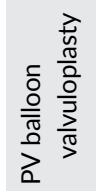

(⿸丆口
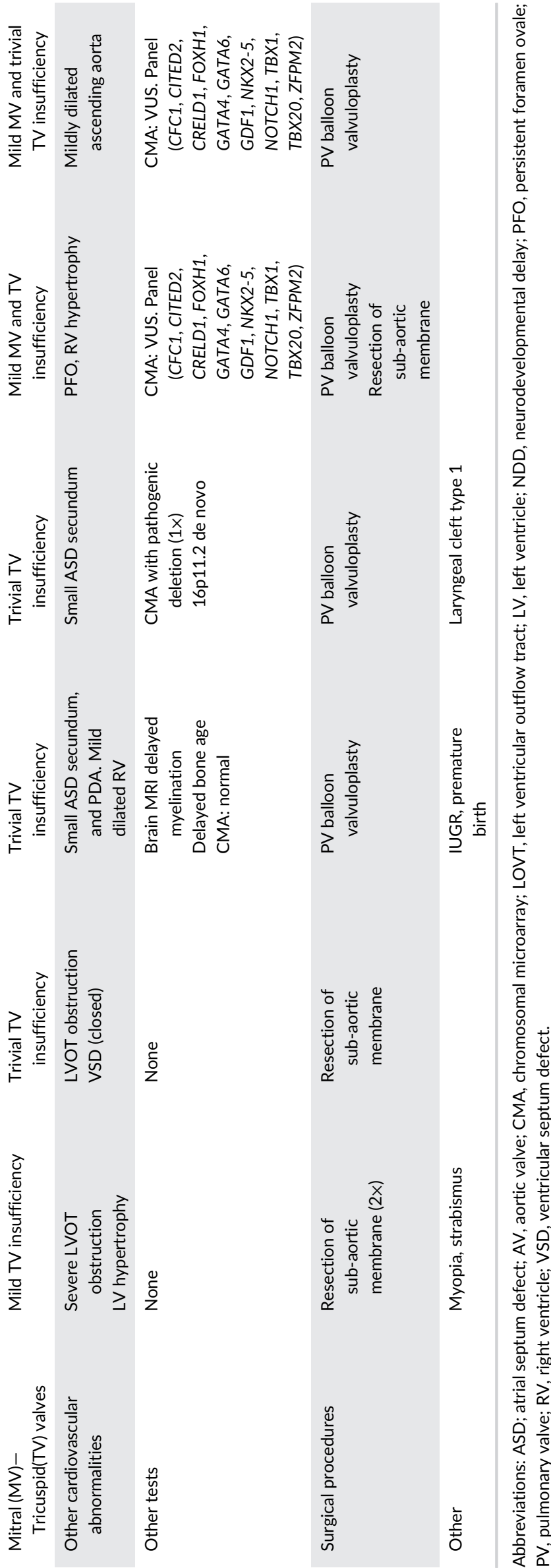

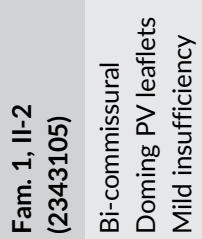
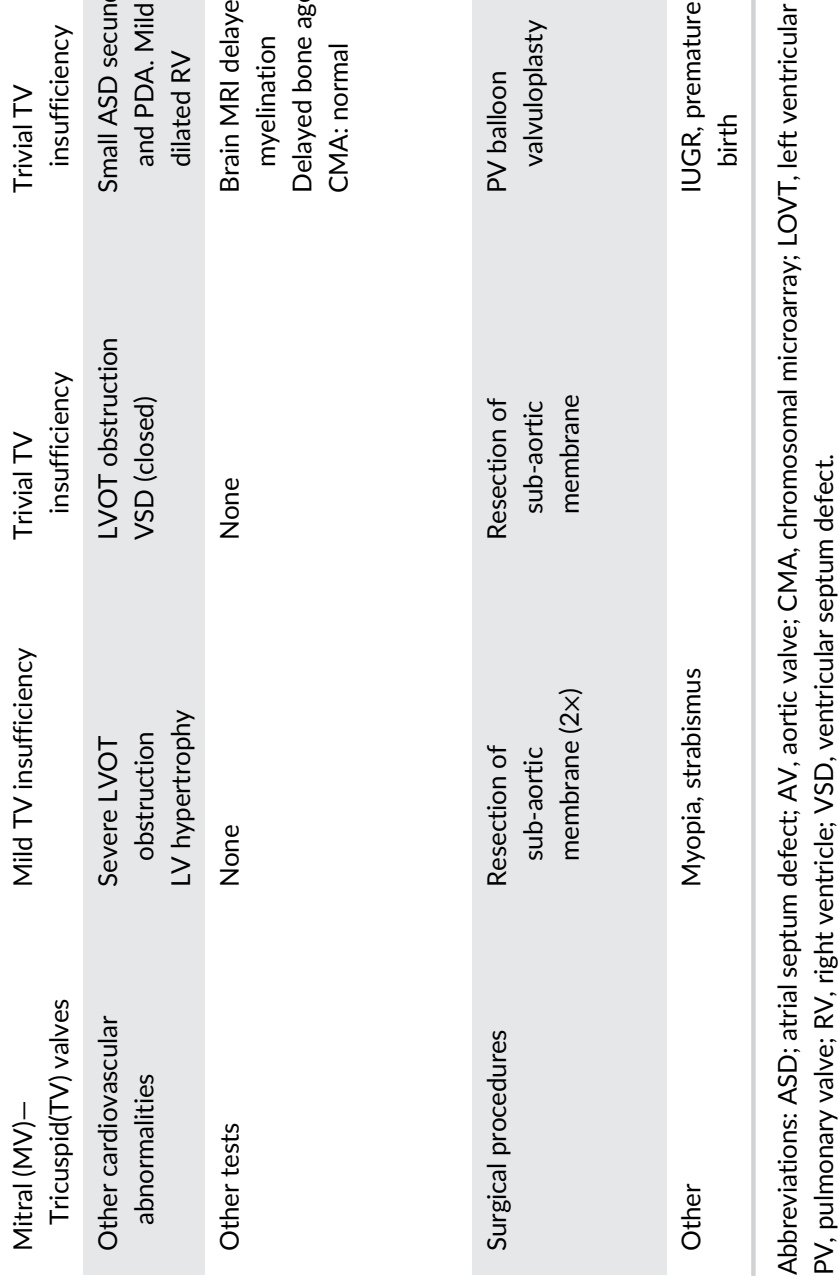
valves. We report three additional LoF variants in six patients from three families and describe the associated phenotype.

In this study, most reported abnormalities include aortic/pulmonary valve thickening, stenosis, and insufficiency. In addition to semilunar valve abnormalities, three out of six patients in our study and one patient described previously ${ }^{10}$ presented with (recurrent) SAM. SAM is a shelf-like membrane that forms below the aortic valve, consisting of several tissue layers including glycosaminoglycans in the subendothelial layer. ${ }^{16}$ SAM results in subaortic stenosis and increased pressure gradient in the LVOT, it has unpredictable hemodynamic progression in childhood and high reoperation rate in up to $34 \%$ of patients. ${ }^{16,17}$ Histopathology of SAM in studied families showed fibromuscular tissue fragments exhibiting secondary regenerative changes on a myxoid background and hypercellularity. These histopathological findings show similarity to those described in Adamts $19^{\mathrm{KO} / \mathrm{KO}}$ mice including hypercellularity, cellular debris, smaller collagen type I fibers and abundant proteoglycans. ${ }^{10}$ It was hypothesized that loss of Adamts19 perturbs shear stress signaling in the endothelial cell leading to increased cellularity and proteoglycan deposition, with increased molecular shear stress signature shown both at scRNA and protein level. ${ }^{10}$ Three out of the six patients reported in this study which had homozygous LoF ADAMTS19 variants, presented SAM with concomitant aortic valve stenosis. SAM reoccurred after surgical resection in two cases, suggesting that in time, hemodynamic factors in combination with loss of ADAMTS19 can lead to SAM recurrence (II-2, family 1 and II-1, family 3). Autosomal recessive and dominant inheritance of SAM was suspected as described occasionally, co-occurring with aortic valve stenosis within families but no gene defect had been recognized to date. ${ }^{18-20}$ ADAMTS19 is the first gene identified related to discrete subaortic stenosis.

In contrast to the reported BAV phenotype in 2/4 patients and the Adamts $19^{\mathrm{KO} / \mathrm{KO}}$ mice by Wünnemann et al, none of the current patients were reported to have BAV. However, one patient presented with bi-commissural pulmonary valve, which was not described in previous cases. Aortic and pulmonary valve stenosis and insufficiency were common features among all patients. In addition to abnormalities of the semilunar valves, $3 / 6$ patients in our study and 2/4 patients from Wünnemann et al also presented with mild involvement of the atrioventricular valves, which contrasted with the absence of atrioventricular valve abnormalities in the Adamts $19^{\mathrm{KO} / \mathrm{KO}}$ mice. Adamts19 is expressed in both semilunar and atrioventricular valves from E14.5 until adulthood in mice. ${ }^{10}$

In general, the phenotype described within this study seems more severe than previously reported, with $2 / 6$ patients presenting with cardiac related complaints. Additionally, while no extracardiac features were described in previous cases, ${ }^{10}$ some of our patients presented with additional features, such as facial dysmorphism (4/6 patients with upslanting palpebral fissures) and delay in language/motor development (2/6 patients, Table 1 ). In one of these patients (II-3, family 2 ), a concomitant $16 \mathrm{p}$ microdeletion (de novo) was detected which explains the neurological phenotype. Given the consanguinity reported in family 2 , we cannot exclude that unidentified variants are causing additional phenotypic features. However, exhaustive exome analysis did not reveal any additional relevant variant.

The cardiac phenotype in our patients and the patients described by Wünnemann et al seems to differ from Notch1-related aortic valve disease: no calcification was observed so far (although all patients are relatively young), no other left side heart defects are present (such as hypoplastic left ventricle) and SAM or subaortic stenosis is never described in Notch-related heart disease. ${ }^{21}$

ADAMTS comprise a family of 19 secreted, extracellular enzymes with a common multidomain structure. The evolutionary conservation of this protein family suggests that they are crucial for mammalian physiology, as shown by the strong phenotypes compromising morphogenesis and relevant functions in humans and mice. ${ }^{22,23}$ As registered in OMIM (accessed January 9, 2020), members of the ADAMTS family are involved in several genetic diseases: Ehlers-Danlos syndrome, dermatosparaxis type (ADAMTS2), Hennekam lymphangiectasia-lymphedema syndrome type 3 (ADAMTS3), Weill-Marchesani syndrome type 1 (ADAMTS10), familial thrombotic thrombocytopenic purpura (ADAMTS13), Weill-Marchesani syndrome type 4 (ADAMTS17) and microcornea, myopic chorioretinal atrophy, and telecanthus (ADAMTS18). Furthermore, based on a recent report, ADAMTS16 might be implicated in LVTO obstruction with extracardiac anomalies ${ }^{24}$ and ADAMTS6 in cardiac conduction defects. ${ }^{25}$

Similarly, Adamts spontaneous and engineered disease models in rodents have been described for 11 Adamts genes. ${ }^{10,23}$ This is not the first time that a gene from this family has been linked to cardiac abnormalities. Kern et al described cardiac and aortic anomalies in adult haploinsuficient mice (Adamts9 ${ }^{+/ L a c z}$ ), probably related to reduced versican cleavage. ${ }^{26}$ Recently, Dupuis et al reported anomalies of the ascending aorta such as increased aortic thickness and aortic stenosis in Adamts 5 deficient mice, probably due to perturbation of Adamts5-mediated cleavage of its proteoglycan substrate aggrecan. ${ }^{27}$ Altogether, these results point toward an important role of several members of the ADAMTS family in cardiovascular health.

\subsection{Limitations of the study}

During this study, we only detected pediatric patients. Further followups and detection of adult patients is needed to better understand the evolution and progression of the ADAMTS19 related disease.

In conclusion, we confirm that LoF variants in ADAMTS19 are causative of a specific cardiac phenotype, characterized mainly by stenosis and insufficiency of the semilunar heart valves. Additionally, the atrioventricular valves are affected in half of the cases, with arterial dilatations occurring occasionally. We conclude that pathogenic ADAMTS19 variants lead to a recognizable cardiac phenotype. Furthermore, we recommend considering ADAMTS19 genetic testing in all patients with multiple semilunar valve abnormalities, particularly in the presence of SAM. This would guide further cardiological screening and follow-ups of the patients and relatives. Finally, ADAMTS19 screening in cohorts of patients with semilunar valve 
abnormalities is needed to estimate the frequency of the HVD related phenotype, which might be not so rare.

\section{ACKNOWLEDGEMENTS}

This work was partially supported by King Abdullah International Medical Research Center through grant RC15/140.

\section{CONFLICT OF INTEREST}

N. O., S. A., S. K., C. B., P. B., A. M. B. A. are employees of CENTOGENE AG. None of the other authors declared a potential conflict of interest.

\section{DATA AVAILABILITY STATEMENT}

The data that support the findings of this study are available from the corresponding author upon reasonable request. Moreover, ADAMTS19 variants described were submitted to the Leiden Open Variation Database (LOVD), with variants ID: 0000663719, 0000663720 and 0000663721

\section{ORCID}

Ingrid M.B.H. van de Laar (D) https://orcid.org/0000-0002-2523-1230

Christian Beetz (D) https://orcid.org/0000-0001-7061-2895

Peter Bauer (D) https://orcid.org/0000-0001-9414-4555

Aida M. Bertoli-Avella (D) https://orcid.org/0000-0001-9544-1877

\section{REFERENCES}

1. Sedmera D. Function and form in the developing cardiovascular system. Cardiovasc Res. 2011;91:252-259.

2. Moorman A, Webb S, Brown NA, Lamers W, Anderson RH. Development of the heart: (1) formation of the cardiac chambers and arterial trunks. Heart. 2003;89:806-814.

3. van der Linde $D$, Konings EE, Slager MA, et al. Birth prevalence of congenital heart disease worldwide: a systematic review and metaanalysis. J Am Coll Cardiol. 2011;58:2241-2247.

4. Hoffman JI, Kaplan S. The incidence of congenital heart disease. J Am Coll Cardiol. 2002;39:1890-1900.

5. Michelena HI, Khanna AD, Mahoney D, et al. Incidence of aortic complications in patients with bicuspid aortic valves. JAMA. 2011;306: 1104-1112.

6. Garg V, Kathiriya IS, Barnes R, et al. GATA4 mutations cause human congenital heart defects and reveal an interaction with TBX5. Nature. 2003;424:443-447.

7. Garg V, Muth AN, Ransom JF, et al. Mutations in NOTCH1 cause aortic valve disease. Nature. 2005;437:270-274.

8. Tan HL, Glen E, Topf A, et al. Nonsynonymous variants in the SMAD6 gene predispose to congenital cardiovascular malformation. Hum Mutat. 2012;33:720-727.

9. Luyckx I, MacCarrick G, Kempers M, et al. Confirmation of the role of pathogenic SMAD6 variants in bicuspid aortic valve-related aortopathy. Eur J Hum Genet. 2019;27:1044-1053.

10. Wunnemann F, Ta-Shma A, Preuss C, et al. Loss of ADAMTS19 causes progressive non-syndromic heart valve disease. Nat Genet. 2019; 52: 40-47.
11. Trujillano D, Bertoli-Avella AM, Kumar Kandaswamy K, et al. Clinical exome sequencing: results from 2819 samples reflecting 1000 families. Eur J Hum Genet. 2017;25:176-182.

12. Bauer P, Kandaswamy KK, Weiss MER, et al. Development of an evidence-based algorithm that optimizes sensitivity and specificity in ES-based diagnostics of a clinically heterogeneous patient population. Genet Med. 2019;21:53-61.

13. Trujillano D, Oprea GE, Schmitz Y, Bertoli-Avella AM, Abou Jamra R, Rolfs A. A comprehensive global genotype-phenotype database for rare diseases. Mol Genet Genomic Med. 2017;5:66-75.

14. Robinson JT, Thorvaldsdóttir H, Winckler W, et al. Integrative genomics viewer. Nat Biotechnol. 2011;29:24-26.

15. Cunningham F, Achuthan $P$, Akanni W, et al. Ensembl 2019. Nucleic Acids Res. 2018;47:D745-D751.

16. Masse DD, Shar JA, Brown KN, Keswani SG, Grande-Allen KJ, Sucosky P. Discrete subaortic stenosis: perspective roadmap to a complex disease. Front Cardiovasc Med. 2018;5:122.

17. Geva A, McMahon CJ, Gauvreau K, Mohammed L, del Nido PJ, Geva T. Risk factors for reoperation after repair of discrete subaortic stenosis in children. J Am Coll Cardiol. 2007;50:1498-1504.

18. Fatimi SH, Ahmad U, Javed MA, Shamim S, Ahmad R. Familial membranous subaortic stenosis: review of familial inheritance patterns and a case report. J Thorac Cardiovasc Surg. 2006;132:1484-1486.

19. Petsas AA, Anastassiades LC, Constantinou EC, Antonopoulos AG. Familial discrete subaortic stenosis. Clin Cardiol. 1998;21:63-65.

20. Piacentini G, Marino B, Digilio MC. Familial recurrence of discrete membranous subaortic stenosis. J Thorac Cardiovasc Surg. 2007;134 818-819. author reply 819 .

21. Kerstjens-Frederikse WS, van de Laar IM, Vos YJ, et al. Cardiovascular malformations caused by NOTCH1 mutations do not keep left: data on 428 probands with left-sided CHD and their families. Genet Med. 2016;18:914-923.

22. Rienks M, Barallobre-Barreiro J, Mayr M. The emerging role of the ADAMTS family in vascular diseases. Circ Res. 2018;123:12791281.

23. Dubail J, Apte SS. Insights on ADAMTS proteases and ADAMTS-like proteins from mammalian genetics. Matrix Biol. 2015;44-46:24-37.

24. Jin SC, Homsy J, Zaidi S, et al. Contribution of rare inherited and de novo variants in 2,871 congenital heart disease probands. Nat Genet. 2017;49:1593-1601.

25. Prins BP, Mead TJ, Brody JA, et al. Exome-chipmeta-analysis identifies novel loci associated with cardiac conduction, including ADAMTS6. Genome Biol. 2018;19:87.

26. Kern CB, Wessels A, McGarity J, et al. Reduced versican cleavage due to Adamts9 haploinsufficiency is associated with cardiac and aortic anomalies. Matrix Biol. 2010;29:304-316.

27. Dupuis LE, Nelson EL, Hozik B, et al. Adamts5(-/-) mice exhibit altered aggrecan proteolytic profiles that correlate with ascending aortic anomalies. Arterioscler Thromb Vasc Biol. 2019;39:2067-2081.

How to cite this article: Massadeh S, Alhashem A, van de Laar IMBH, et al. ADAMTS19-associated heart valve defects: Novel genetic variants consolidating a recognizable cardiac phenotype. Clin Genet. 2020;1-8. https://doi.org/10.1111/ cge. 13760 